\title{
A Simplified Procedure for the Seismic Design of Hybrid Connections in Precast Concrete Structures
}

\author{
F. Porco*, D. Raffaele and G. Uva
}

Dipartimento DICATECh, Politecnico di Bari, Italy

\begin{abstract}
The correct use of non-adherent prestressing techniques in beam-column connections can significantly increase the seismic performance of precast concrete frames, making them a competitive alternative to the traditional cast-in-place concrete structures. In this sense, a fundamental factor of the nodes' design is represented by the correct calibration of the ratio between the amount of mild steel and that of post-tensioned reinforcements, in order to provide the prescribed flexional and rotational capacity to the beam-column interface, and to guarantee at the same time the required dissipative and re-centering capacity. In this paper, a simple algorithm for the optimal and quick pre-dimensioning of the above mentioned parameters is proposed. It is based on the knowledge of the materials' mechanical parameters and of the "target" stresses and deformations corresponding to the required performance level, which can be computed either through a Force Based Design approach or by a Displacement Based Design approach.

In the case of centered post-tension and in the presence of symmetrical mild steel, the procedure can also be effectively represented in a graphical form, making it is easily possible to determine the post-tensioning stress that should be applied to the cables in order to guarantee that they remain in the elastic field after the seismic event, and to obtain the recentering of the system.
\end{abstract}

Keywords: Precast concrete frames, hybrid beam-column connections, post-tension, self-centering capacity, seismic design.

\section{INTRODUCTION}

Over the last thirty years, the scientific community has been extensively involved in research programs aimed at investigating the possibility of an organic approach to the use of precast components, in order to overcome the traditional idea that their design should "emulate" that of cast-in-place structures. Still today, in fact, the advantages of using prefabricated components also in areas of high seismicity are not yet fully exploited because of the restrictive prescriptions contained in many international technical codes $[1,2]$. In these codes, as a matter of fact, the use of precast systems in which elements are assembled by using ductile dry connections is limited, and preferably the use of integrative cast-in-place concrete filling , "wet" connections, is suggested, which of course increases execution time and related costs.

The recent ACI standards [3] contain design provisions for precast concrete structures exposed to high seismic risk.

It is anyway pointed out that such requirements are based predominantly on field and laboratory experience with monolithic reinforced concrete building structures and precast concrete building structures designed and detailed to behave monolithic. The extrapolation of these requirements to other types of cast-in-place or precast concrete structures should be based on evidence provided by field experience, tests, or analysis.

*Address correspondence to this author at the Dipartimento DICATECh, Politecnico di Bari, via Orabona 4, 70126 Bari, Italy; Tel: +39 080 5963832; Fax: +39 080 5963832; Email: f.porco@poliba.it
In the last few years, the use of post-tensioning techniques for assembling precast elements has been considered with great interest. Many experimental studies were devoted to this issue, which however showed some drawbacks: these systems have a very low energy dissipation if compared to wet connections, and, besides, they exhibit a relevant stiffness reduction because of the pretension losses suffered by the tendons.

The inspiration of introducing unbonded tendons in the connection among precast beams and columns in order to optimize the seismic performance of the system was proposed for the first time by Priestley and Tao [4]. Later, Stone and others [5] drew on this initial idea, while experimental evidences were provided by the PRESSS (PREcast Seismic Structural System) research program, carried out at UCSD (California University, San Diego) from 1990 to 1999 [6]. During this program extensive experimental tests were performed over models of a 5 storey building (in a 2:3 scale) subjected to seismic loads, providing different types of nodes among the precast elements, both for moment frames and shear walls resisting systems.

By now, it has been widely demonstrated through both experimental and theoretical studies that the use of unbonded prestressing tendons as a connection system significantly improves the seismic performance of precast frames, that can so be conveniently used as a competitive option to cast-inplace frames.

It is important to emphasize that a widespread use of precast systems, with elements made in the factory, would avoid the dispersion of the resistance values of the in situ concrete 
that often adversely affects the structural performance [7-9].

In the paper the peculiar features of systems based on hybrid nodes will be extensively discussed.

The aim is that of describing a method for the rapid optimal preliminary design of the main parameters: posttensiones cables area, initial value of the pretension and mild steel area.

With this procedure it is possible to attain the required flexural rotational and dissipative capacity as well as the self-centering capacity, that is the ability of the structure to return towards its initial configuration upon unloading.

\section{SEISMIC PERFORMANCE OF DUCTILE CON- NECTIONS}

\subsection{Traditional Prestressed Connections}

Precast frames assembled using traditional prestressing techniques, i.e. adherence is obtained by grouting the cables after post-tension, have generally shown a good level of ductility, which is comparable to that of traditional cast-in-place frames. Nevertheless, even for low damage levels, these frames exhibit an excessive and dangerous stiffness reduction $[10,11]$, which will determine a drop of the original pretension level when the initial strain configuration is recovered after the seismic event. The higher the excursion beyond the proportionality threshold during the earthquake, the more severe the loss of the prestress is.

For example, in the case of the connection shown in (Fig. 1a), this phenomenon would considerably reduce the stiffness of the system, consequently reducing the capacity to support vertical loads, and increasing the amount of shear reinforcement required to guarantee the proper flexional capacity for the beam and for the node [12]. The phenomenon is described in the diagram of (Fig. 1b), showing the stressstrain diagram of the prestressing cable $\left(\sigma_{s p}-\varepsilon_{s p}\right)$.

If $\sigma_{s p 1}$ is the service stress in the cable, purged of the initial losses of tension, under a small variation around point 1 the cable remains within the elastic field, and no stress drop is produced. Under higher seismic loads, instead, the struc- ture enters in the plastic field. Let's suppose that the cable reaches the stress-strain state represented by the point 2 in (Fig. 1b), belonging to the plastic branch of the constitutive curve. It can be immediately observed that the unloading path to the initial deformation $\varepsilon_{s p 0}$ is characterized by the pretension loss $\left(\sigma_{s p 1}-\sigma_{s p 2}\right) \cdot A_{p}$. It could be also happen, for higher ductility levels (point 3 in Fig. 1b), that the whole initial pretension force is lost.

In conclusion, a most serious risk is that the loss of pretension induced by the yielding of the prestressing cables can make the system unable to sustain even the only gravity loads.

\subsection{Prestressed Connections Realized with Post- Tensioned Unbonded Tendons}

The use of unbonded cables presents a number of advantages, which were highlighted also by experimental research programs, among which it is worth mentioning the work carried out by Priestley and Tao in 1993 [4] on node prototypes like the one shown in (Fig. 2a), where the assembly of precast beam-column elements was obtained by using unbonded cables designed in order to maintain an elastic behavior throughout the load cycle.

Among the features characterizing the seismic performance of this type of nodes, the following aspects can be pointed out:

a) Since the behavior of the cable is purely elastic even under the maximum displacement demand, seismic actions will not induce any loss of prestress, and therefore the frictional shear resistance of the interface section - and the related resistance to vertical loads - will not be permanently affected at the end of the horizontal load cycle.

b) This type of connection cables are accommodated into post-tensioning ducts without any grouting, and this has the non-negligible advantage of dramatically reducing the seismic damage level - which is typically concentrated at the interface sections - whereas in other node types, because of the adherence, it is usually spread all over the structural components. In other words, this involves a significant reduction of repair and maintenance costs and, more in general, a mitigation of the conse-

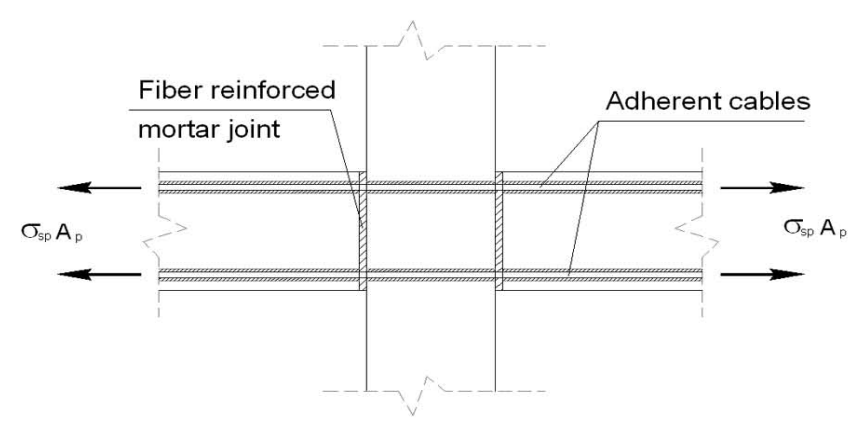

(a)

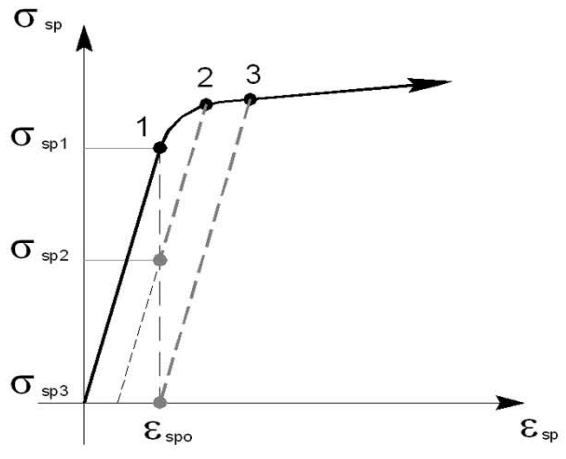

(b)

Fig. (1). (a) Beam-column connection realized with adherent cables; (b) Stress-strain diagram for the prestressing cable. 
quences related to the possible interruption of use and loss of serviceability.

c) The overall system's structural response is basically elastic, even if non-linear. When considering the seismic performance, this behavior has the advantage of allowing the system to recover the initial undeformed configuration, with no appreciable residual deformation after the earthquake. On the other hand, this means also that there is a almost no energy dissipation capacity (Fig. 2b), and that the strength demand will consequently be high.

Fig. (2b) describes the typical force displacement relationship $(F-\Delta)$ for the connection type shown in (Fig. 2a), realized through post-tensioned unbonded cables. In the diagram, $\mathrm{F}$ and $\Delta$ respectively are the force applied and the horizontal displacement of the characteristic point chosen for monitoring the system. The beam-column interface section is fully compressed and the overall response of the node is linearly elastic until point 1 , corresponding to the first cracking of the concrete.

With a good approximation, a linear elastic behavior can be assumed until the crack is limited to half the height of the beam (point 2 in the diagram of Fig. 2b). Beyond this point, the force-displacement relationship is a highly non-linear elastic one, and finally, at point 3 the cable stress reaches the yielding limit.

\subsection{Hybrid Connections}

The main disadvantage of the post-tensioned cable connections described in the previous paragraph is represented by the non-linear elastic response of the system, that is virtually associated with a zero additional damping. Consequently, a very low dissipation capability can be attained and a higher demand of structural strength is required.

The seismic behavior of prestressed connections with unbonded cables can be considerably improved by the implementation of the so-called "hybrid connection", obtained by combining the use of unbonded tendons with the simultaneous use of mild reinforcement [5].

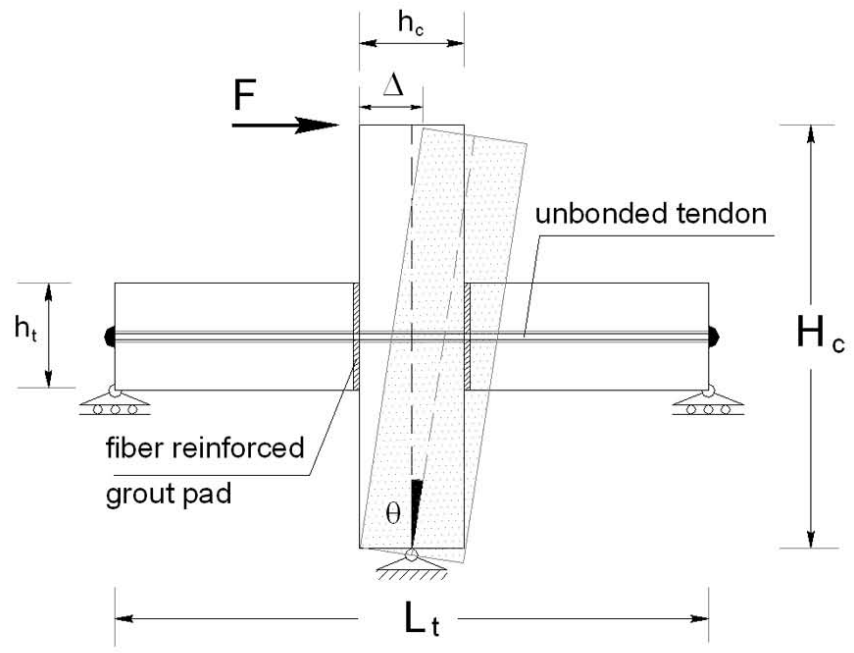

(a)
As well as the classical prestressed nodes, such a connection (Fig. 3) is conceived in order to lump the damage distribution at the interface section, thanks to the opening/closing effect, but allows at the same time a significant energy dissipation related to the yielding of the ordinary reinforcements. Also in this case, the cables keep an elastic behavior during the seismic event, and possible losses of prestress are prevented. Consequently, the frictional shear resistance at the interface is kept at the maximum level, and a significant selfcentering capacity is guaranteed, minimizing possible residual deformations.

The characteristic Force-Displacement curve of a hybrid connection is substantially different from that of conventional prestressed nodes.

Fig. (4a) shows, in a simplified bi-linear form, the typical non-linear elastic response of a connection consisting only of unbonded post-tensioned cables, obtained by plotting the bending moment $\mathrm{M}$ applied at the node against the rotation $\theta$, as defined in (Fig. 2). The hypothesis of elasticity means that, during the loading and unloading cycles, the same initial path is always followed, and that no energy is dissipated.

On the other hand, (Fig. 4b) shows the ideal hysteretic cycle of an elastic-plastic system endowed with energy dissipation. When loads are removed, this system can present a consistent residual displacement, usually comparable to the maximum shift attained during the loading cycle.

Obviously, hybrid systems are characterised by a combination of the previously described behaviors.

A particularly effective solution is shown in (Fig. 4c) (flag shape hysteresis loop): the dissipative capacity typical of mild reinforcements is combined with the self-centering properties of unbonded cables in such a way that the system has no residual displacement when the load is removed [4].

As a limit case, in the presence only of the unbonded post-tensioned cables, the constitutive behavior would be the non-linear elastic one shown in (Fig. 4a), which guarantees the complete self-centering of the system. If the unbonded 

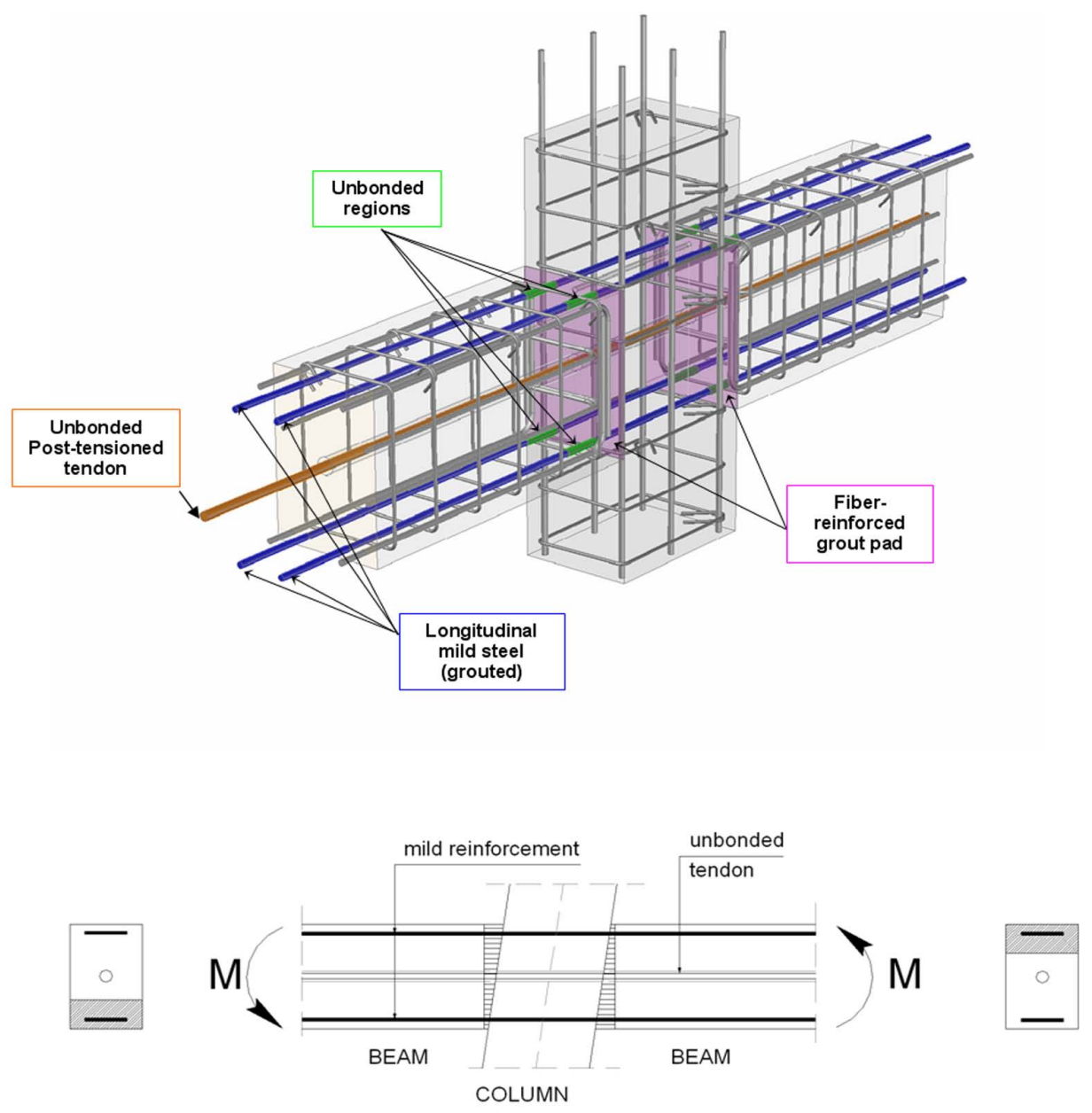

Fig. (3). Schematization of a Hybrid beam-column connection [13].
Flexural contribution
Flexural contribution
of unbonded tendon
of mild steel

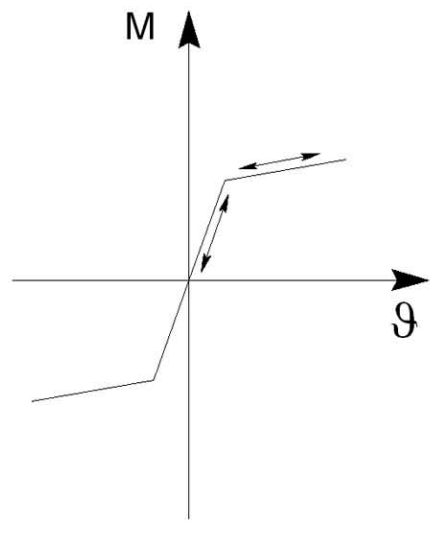

Self-centering

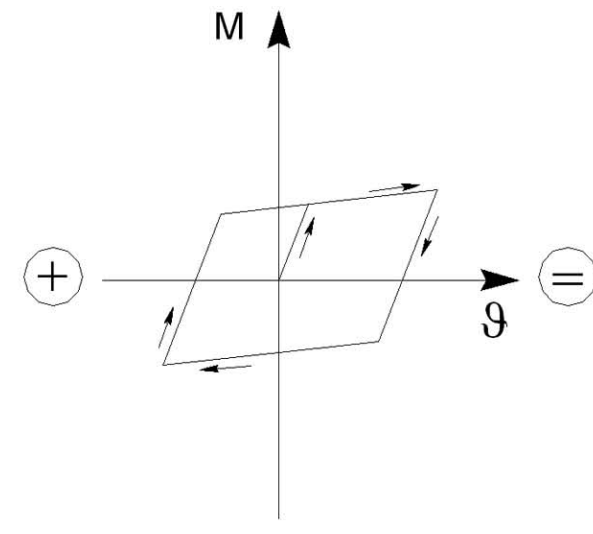

Energy dissipation

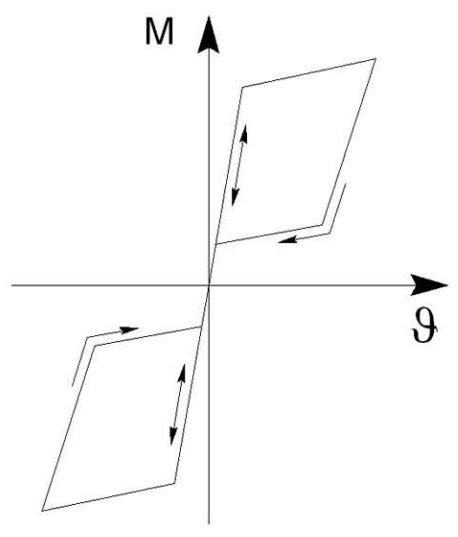

Hybrid system

Fig. (4). Typical bending moment-rotation curves: a) for a bi-linear elastic system; b) for a hysteretic dissipative system; c) for a hybrid system.

tendons are missing, instead, the behavior is characterized by the hysteretic cycle shown in (Fig. 4b), which allows the maximum available energetic dissipation, but requires as well a great residual plastic deformation. It can be observed that if the contribution of the post-tensioned cables $\left(M_{r p t}\right)$ is increased, a lower dissipative capacity results, but the system can provide a higher displacement demand, thanks to a greater self-centering capacity. 
On the other hand, by reducing the amount of posttensioned steel, the dissipative capacity grows, whereas residual displacements becomes relevant, with a consequent increase of both damages and related costs for the postearthquake retrofitting.

The optimal balance between $M_{r p t}$ and $M_{s t}$ is usually deduced from the required performance level, that is to say by controlling the maximum displacement expected under the design earthquake and the acceptable damage, expressed by a proper limitation of residual deformations.

Thence, once the final flexural capacity $\mathrm{M}_{\mathrm{rd}}$ of the node is fixed, the flexural contribution $\mathrm{M}_{\mathrm{rpt}}$ of the post-tensioned cables and the contribution $\mathrm{M}_{\mathrm{rs}}$ of the yielded mild steel can be properly combined and used as the main design parameters.

The preliminary design of hybrid connections will then provide, first of all the a-priori choice of the parameter $\Gamma$ :

$$
\Gamma=\frac{M_{r p t}}{M_{r d}}
$$

According to the previous considerations.

Then, according to the scheme of (Fig. 5), a reliable value of the equivalent damping of the system $\left(\xi_{e q}\right)$ can be determined, or, alternatively, a proper behavior factor $q$. The first parameter is the basis for the definition the secant stiffness of the equivalent system, if a Displacement Based Design approach is to be used, whereas the second one is useful for a classical Force Based Design.

The diagrams show that by increasing the contribution related to the post-tension, the dissipative capacity is reduced, whereas the system is still able to satisfy the peak displacement demand, thanks to the higher self-centering capacity.

On the other hand, by reducing the amount of posttensioned reinforcements, the dissipative capacity is increased but the residual drift becomes non-negligible, with a considerable growth of the seismic damage level and of the consequent repair and maintenance costs after the earthquake.

For these structural types, a correct design should therefore require the a-priori definition of the contribution to the overall flexural node's resistance respectively assigned to the yielded mild steel $\left(M_{r s}\right)$ and to the prestressing cables $\left(M_{r p t}\right)$. In particular, the preliminary knowledge of $\Gamma$ ratio will allow to define a reliable value of the equivalent damping ratio $\xi_{\text {eq }}$, which is the essential element to be used either in the evaluation of the secant stiffness of the "replacement system" within a Direct Displacement Based Design (DDBD, [11]), or in the determination of the adequate behavior factor $q$, according to the classical Force Based Design approach - FBD (see Fig. 5).

The design of the reinforcements, according to the choice made about $\Gamma=\mathrm{M}_{\mathrm{rpt}} / \mathrm{M}_{\mathrm{rd}}$ will then follow from these parameters, and it will be also possible to check if during the loading process the stress level of the prestressing cables remains within the elastic limits and if the connections keep an adequate self-centering capacity. If the selected ratio $\Gamma$ does not satisfy the design performance level, it will be necessary to recalibrate it. This will require the identification of a new value of equivalent viscous damping and the reiteration of the related DDBD process, or alternatively the redefinition of the structural factor q, preliminary to a new FBD analysis.

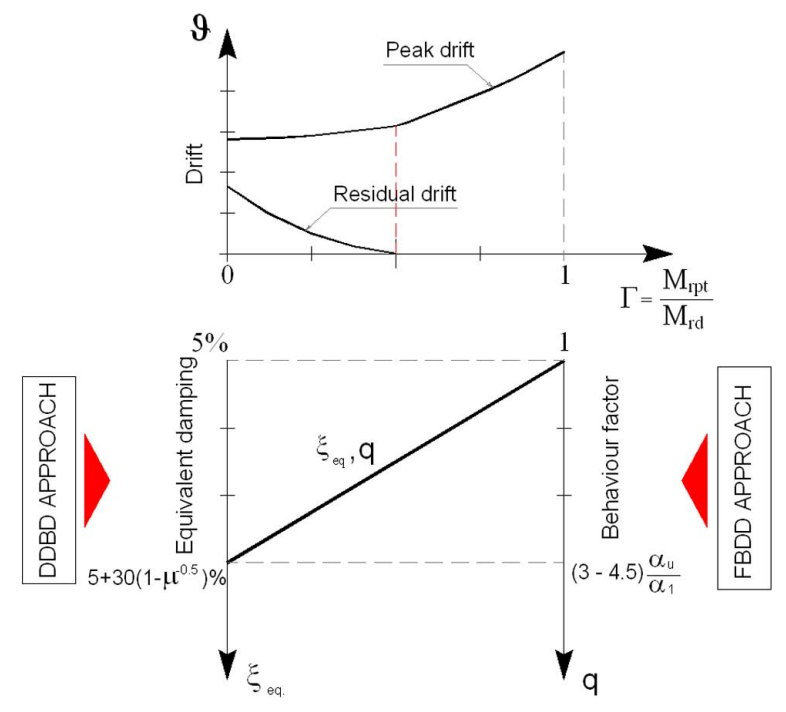

Fig. (5). Diagrams showing the variation of the drift $\theta$ of the node (top), equivalent damping and structural factor (bottom) with the parameter $\Gamma=M_{r p t} / M_{r d}$.

\section{A SIMPLIFIED PROCEDURE FOR THE DESIGN OF HYBRID CONNECTIONS}

\subsection{Parameters and Equations Governing the Design}

Precast concrete frames are often introduced in buildings with the sole purpose of operating as a bracing element, whereby the effects of gravity loads become negligible if compared to seismic stress, and the adoption of symmetrical mild reinforcements together with a centered post-tension results particularly rational. In this case, the design of the beam-column interface section of a hybrid connection can be conveniently performed by means of the simple approach that is based on the use of few dimensionless parameters which express the main variables involved in the analysis (Fig. 6) [14].

The following symbols and non-dimensional parameters are now introduced:

$$
\begin{aligned}
& \mathrm{b}=\text { depth of section } \\
& \mathrm{d}=\text { effective height of section } \\
& \xi=x / d=\text { normalised neutral axis depth } \\
& \lambda=c / d=\text { normalized concrete cover depth } \\
& \rho_{s}=A_{s} / d=\text { mild reinforcement ratio; } \\
& \psi_{s}=f_{y s} / f_{c} \\
& f_{c}=\text { concrete compressive strength } \\
& f_{y s}=\text { nominal yield strength of steel; }
\end{aligned}
$$




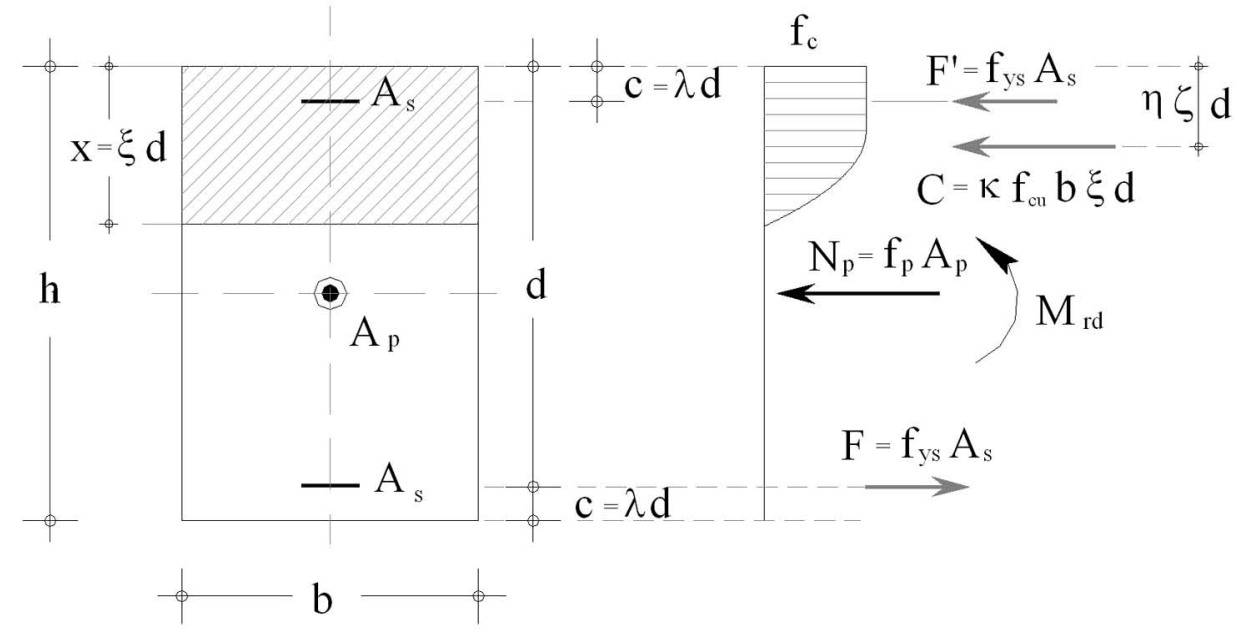

Fig. (6). The Beam-column interface section: geometry; definitions and symbols; stress distribution.

When the mild steel is yielded, the resistant moment $M_{r d}$ of the section can be expressed in dimensionless terms by the following equation:

$$
m_{r d}=\frac{M_{r d}}{b d^{2} f_{c}}=-\kappa\left(\eta \xi^{2}-\xi \frac{1+\lambda}{2}\right)+2 \rho_{s} \psi_{s} \frac{1-\lambda}{2}
$$

Similarly, the dimensionless expression of the axial force $N_{p}$ (which in this case is given by the post-tension in the cable) is:

$$
n_{p}=\frac{N_{p}}{b d f_{c}}=\kappa \xi
$$

The value of the constants $\kappa$ and $\eta$, which defines the resultant of the compressive stresses over the section and the position of the neutral axis depends on the constitutive law adopted for the concrete.

In (Fig. 7), $\theta$ and $M_{r d}$ respectively are the deformation measure and the internal action of a typical node obtained after a DDBD/ FBD analysis of the overall structure, in which it has been assigned in advance the value of $\Gamma=M_{r p t} / M_{r d}$, flexural capacity provided by the post-tension cable over total flexural capacity of the beam-column interface at the target deformation.

From the analysis of equation (2), it can be noticed that the first term at the right member represents the contribution of the cable stress to the non-dimensional resistant moment $m_{r p t}$, whereas the second term defines the quota $m_{r s}$ took over by the mild steel, which is supposed to be yielded in correspondence of the actual limit deformation.

It is worth observing that the target drift plays a fundamental role in defining the amount of post-tension reinforcement whose behavior must remain within the proportionality limits under this deformation, if we wish to avoid dangerous losses of prestress. Thence, it is fundamental to identify first of all the relationship that links the flexional capacity $m_{r p t}$ supplied by the cable to the rotation $\theta$ at the beam-column interface.

With the notations in (Fig. 7), if the cable is supposed to be unbonded along the entire length $L_{n a}$, it is possible to ex- press the cable elongation as a function of the rotation $\theta-$ or equivalently as a function of the strain variation $\Delta \varepsilon_{p}$ of the cable - as follows:

$$
\Delta L=2 \vartheta \cdot\left(\frac{h_{t}}{2}-x\right)=\Delta \varepsilon_{p} \cdot L_{n a}
$$

that, if we call $\tau_{n a}$ the length of the unbonded tendon belonging to each beam-column interface (in the case of the figure: $\tau_{n a}=L_{n a} / 2$ ), becomes:

$$
\vartheta \cdot\left(\frac{h_{t}}{2}-x\right)=\Delta \varepsilon_{p} \cdot \tau_{n a}
$$

By introducing the parameter $\chi_{\mathrm{t}}=\tau_{n a} / h_{t}$, representing the ratio between the portions of the cable previously defined and the overall height of the section $h_{t}$ (see Fig. 7), and the non-dimensional measures of the parameters shown in (Fig. 6), the additional specific elongation $\Delta \varepsilon_{p}$ of the cable can also be written as:

$$
\Delta \varepsilon_{p}=\frac{\vartheta}{\chi_{t}}\left(0.5-\frac{\xi}{1+\lambda}\right)
$$

From eqs. (5) and (6), the relationship between the interface rotation $\theta$ and the cable elongation at the limit strain is finally obtained:

$$
\vartheta=\frac{\chi_{t}}{0.5-\frac{\xi}{1+\lambda}} \Delta \varepsilon_{p}
$$

For the design purpose, it is supposed that the cable has just been reached, with no further excursion, the limit elastic deformation, and thence the corresponding stress value $f_{p y}$.

Consequently the strain variation of the cable is:

$$
\Delta \varepsilon_{p}=\frac{f_{p y}-f_{p i}}{E_{s}}
$$

where $f_{p i}$ is the initial stress applied to put the cable under tension (net of the initial loss). 


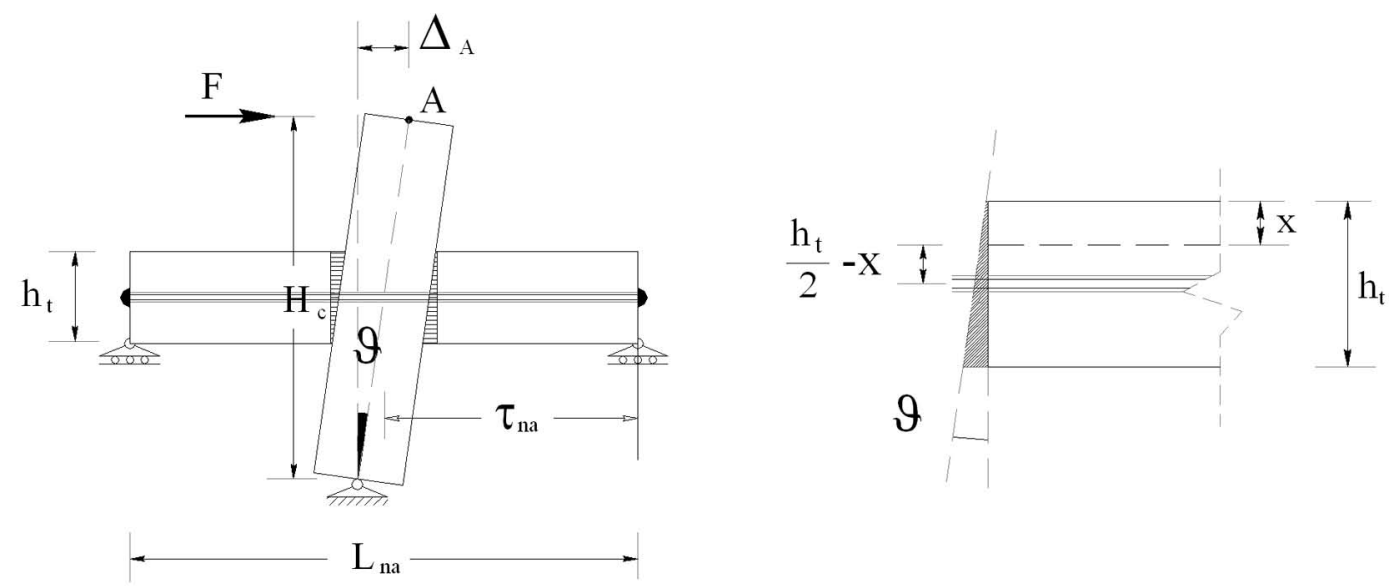

Fig. (7). Deformation at the beam-column interface in correspondence of the target drift.

After introducing the non-dimensional quantities:

$$
\begin{aligned}
& \psi_{p y}=\frac{f_{p y}}{f_{c}}, \\
& \psi_{p i}=\frac{f_{p i}}{f_{c}}, \\
& \psi_{E}=\frac{E_{s}}{f_{c}}
\end{aligned}
$$

and defining:

$$
\beta=\chi_{t} \cdot\left(\frac{\psi_{p y}-\psi_{p i}}{\psi_{E}}\right)
$$

Eq. (7) can also be written as:

$$
\vartheta=\frac{\beta}{0.5-\frac{\xi}{1+\lambda}}
$$

Using the previous results, and after some algebraic manipulation of eq. (2), the following expression for $m_{r p t}$ can be deduced:

$$
m_{r p t}=-\kappa\left(\eta \xi^{2}-\frac{\xi}{2}(1+\lambda)\right)
$$

Solving eq. (9) for $\xi$, and replacing it in (10), it is finally possible to obtain the function expressing the contribution $m_{r p t}$ of the cable, that supposed to work at the elastic proportionality limit, and the rotation $\vartheta$, which is assumed as the main performance design parameter:

$$
m_{r p t}=-\kappa(1+\lambda)^{2} \frac{\vartheta-2 \beta}{4 \vartheta^{2}}[\vartheta(\eta-1)-2 \eta \beta]
$$

In (Fig. 8a) it is shown the diagram of the function $m_{r p t}(\theta)$, according to eq. (11), whereas in (Fig. 8b) the variation of $m_{r p t}$ and $\theta$ with the position of the neutral axis is shown. All the diagrams are presented for a set of values of the factor $\beta$, which depends on the concrete class and the steel type, obtaining a set of curves. Besides, the diagrams were plotted assuming the parabola-rectangle constitutive law provided by the technical standards for concrete classes with $f_{c k} \leq 50 \mathrm{MPa}[1,2]$, assuming the values $\kappa=0.809 ; \eta=0.416$.

\subsection{Some Remarks}

With reference to the diagrams of (Fig. 8), when a couple $\left(\mathrm{m}_{\mathrm{rpt}}=\Gamma \mathrm{m}_{\mathrm{rd}} ; \theta\right)$ corresponding to the performance required to the node is assigned, it is immediately singled out the value $\bar{\beta}$, and, consequently, the maximum post-tension value for which the cable remains within the elastic field during the seismic event.

In fact, remembering the expression of $\beta$, we obtain:

$$
\left(\frac{\psi_{p i}}{\psi_{s}}\right)_{\max }=\frac{\psi_{p y}}{\psi_{s}}-\frac{\psi_{E}}{\psi_{s}} \frac{\bar{\beta}}{\chi_{t}}
$$

When the limit resistance of the section is attained, the maximum compressive force in the concrete must be equal to the maximum force exerted by the tendon at the elastic limit, since the mild reinforced have been supposed to be symmetrical and are both yielded. This implies:

$$
\kappa \cdot f_{c} \cdot b d \cdot \xi=A_{p} \cdot f_{p y}
$$

from which, called $\rho_{p}=A_{p} / b d$, we obtain:

$$
\xi=\rho_{p} \frac{\psi_{p y}}{\kappa}
$$

This relationship provides the amount of steel required for the post-tensioning as a function of the position reached by the neutral axis at the yielding limit of the post-tensioned reinforcements.

It should be noted that the design of the prestressing reinforcement is independent both from the mild steel amount and from the initial value of the pretension force, whose maximum value is governed by eq. (11).

As previously remarked, the $2^{\text {nd }}$ addendum at the right member of eq. (2) represents the contribution $m_{r s}$ to the total flexural capacity of the section provided by the yielded mild 

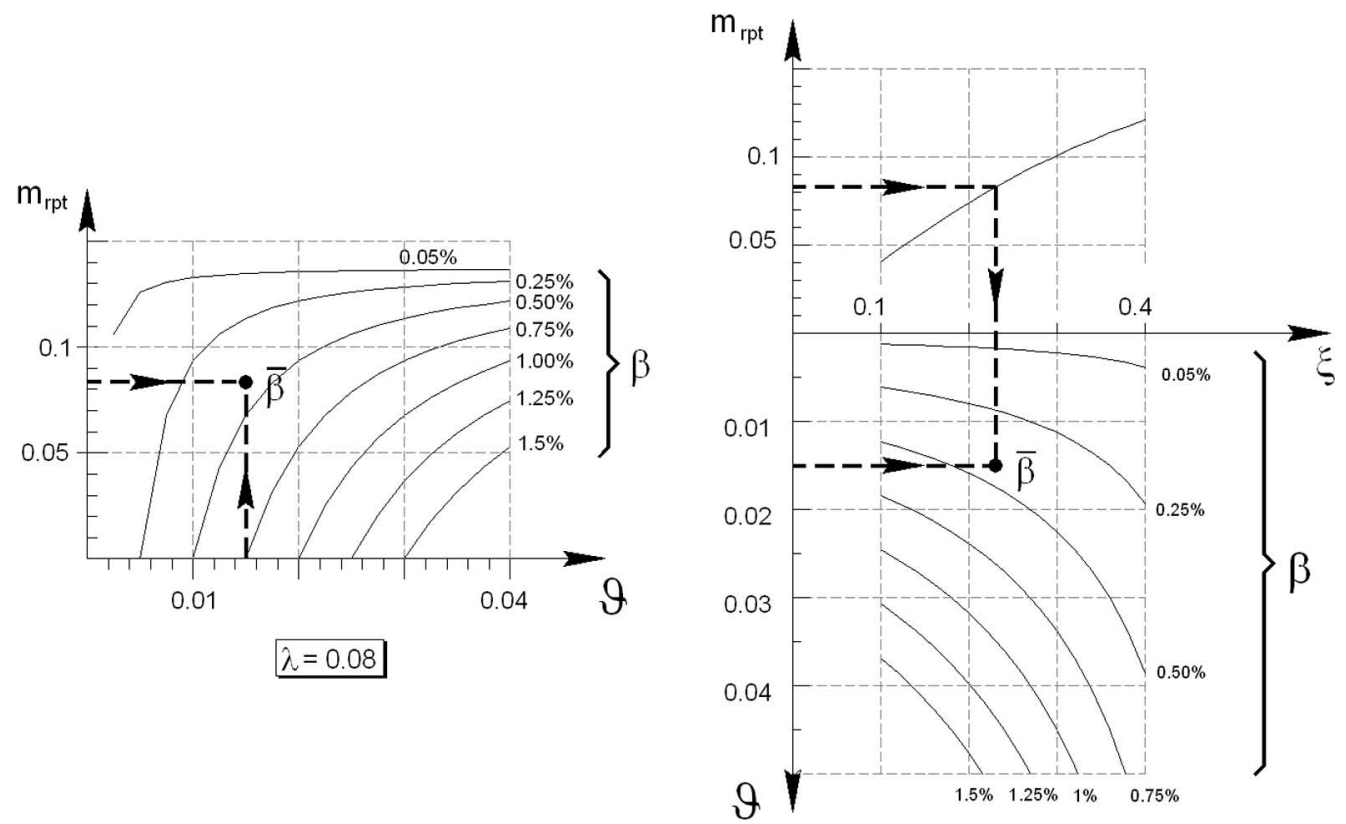

Fig. (8). (a) $m_{r p t}-\vartheta$ diagram for different values of $\beta$. (b) Plot of the functions $m_{r p t}(\xi)$ and $\vartheta(\xi)$ for different values of $\beta$.

steel. Remembering that $\Gamma=M_{r p t} / M_{r d}$, this can be also written as:

$$
m_{r s}=\rho_{s} \psi_{s}(1-\lambda)=m_{r p t} \frac{1-\Gamma}{\Gamma}
$$

Another fundamental aspect in the design of hybrid nodes is represented by the monitoring of the self-centering capacity of the connections.

It should be noted that, when the external actions cease, all the mild steel will be compressed and, therefore, in order to ensure the self-centering of the section it will be sufficient that the prestressing cables provide a tensile action greater then the compression resultant of the ordinary reinforcement. In formulas, this means that it should result:

$$
\rho_{p} \cdot f_{p i} \geq 2 \rho_{s} \cdot f_{s y}
$$

which, with the usual non-dimensional notations, can be written as:

$$
\rho_{s} \leq \frac{1}{2} \rho_{p} \frac{\psi_{p i}}{\psi_{s}}
$$

At last, it should be pointed out that if the condition expressed by eq. (17) is not satisfied, it is necessary to restart the design of the section, by increasing the parameter $\Gamma$. This obviously means that the new equilibrium condition for the resistant moments at the node also changes the individual value of the basic design parameters, the equivalent viscous damping ratio $\xi_{\text {eq }}$ in a DDBD or the behavior factor $q$ in an FDB, and the entire analysis must be restarted by using new value of the parameter $\Gamma$.

\subsection{An Iterative Simplified Procedure for the Prelimi- nary Design Based on a 4-Way Abacus}

The observations presented in the previous paragraphs are an useful basis for the design of an hybrid connection, and allow to address the procedure to a particularly convenient and simple iterative approach, summarised in the flow chart shown in (Fig. 9) and briefly explained below:

a. First of all, a trial value is set for the parameters $\xi$ (defining the position of the neutral axis) and $f_{p i}$, (which represents the initial tensile force applied to the tendon, after deducing the initial losses).

b. From eq. (6), the value of the additional specific elongation of the cable is computed as a function of the design rotation $\theta$ of the connection, and the overall force is thence known: $f_{p}=f_{p i}+\Delta \varepsilon_{p} E_{s}$.

c. A check is carried out to see whether the cable has reached the yielding point and, if so, the initial pretension force is reduced, returning to step 1.

d. According to the values chosen for the neutral axis position and for the parameter $\Gamma$, on which the analysis is based, the two contributions $m_{r p t}$ and $m_{r s}$ of the resistant moment of the interface section are calculated, together with the corresponding steel ratio: $\rho_{s}=m_{r s} /(1-\lambda) \psi_{s}$ and $\rho_{p}=\kappa \xi / \psi_{p}$.

e. It is checked if the resultant flexural capacity $M_{d}$ satisfies the condition $m_{d} \geq m_{r p t}+m_{r s}$, and if it doesn't, the position $\xi$ of the neutral axis is changed, coming back to the step 2 of the procedure and restarting the dimensioning of the reinforcements.

f. At the end, after a positive feedback about the previous point, the existence of an adequate self-centering capacity of the section is checked $\left(\rho_{p} \geq 2 \rho_{s} \cdot \psi_{s} / \psi_{p i}\right)$, otherwise the parameter $\Gamma$ is reconsidered, by increasing the contribution $m_{r p t}$ of the cable and restarting from step 1 .

After completing the above described procedure, it is highly advisable to assess the compatibility of the maximum strains attained in the mild reinforcement by considering the 


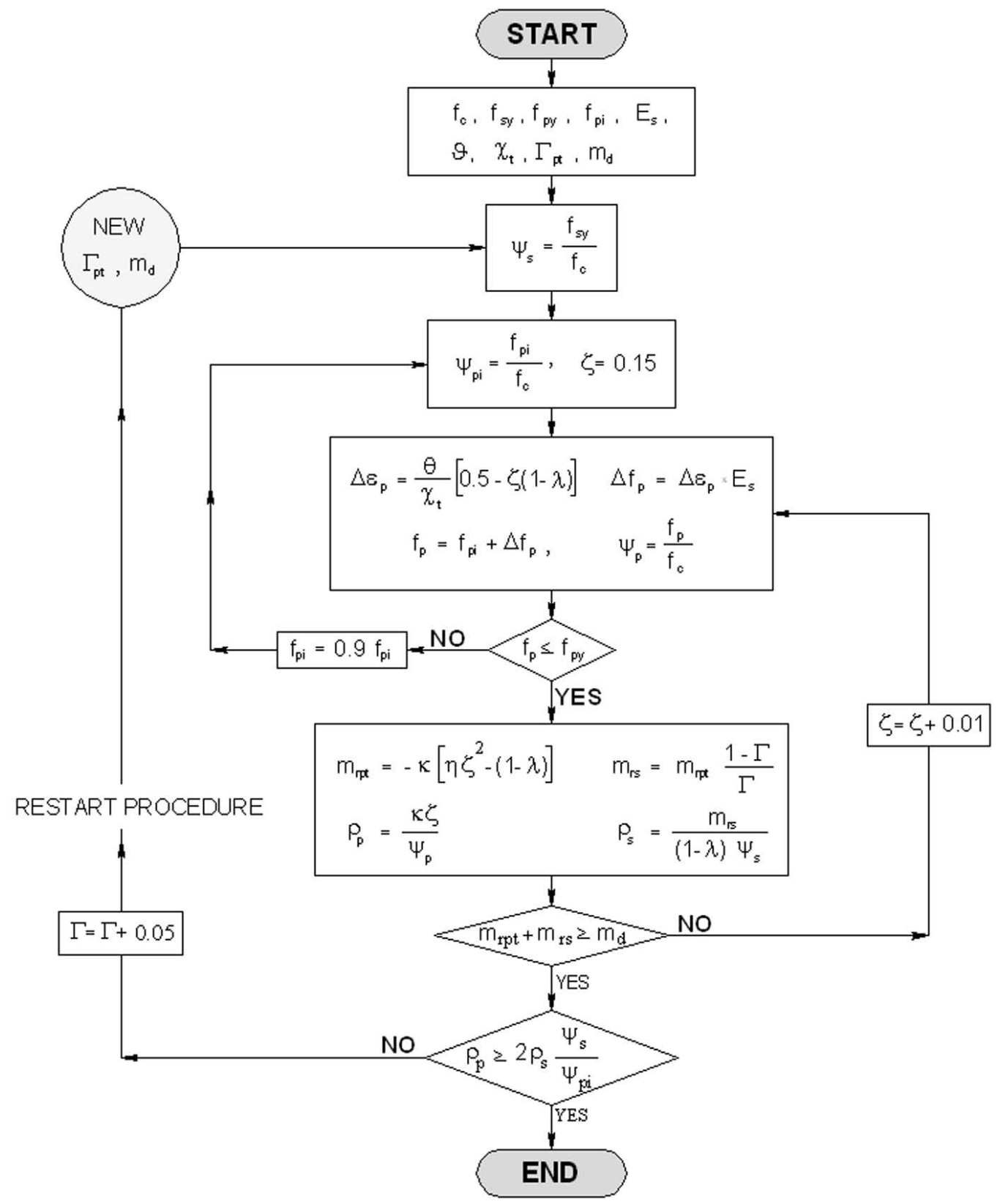

Fig. (9). Flow chart of the procedure for the design of hybrid connections.

actual mechanical parameters of the steel, above all with respect to a possible fatigue behavior.

An aspect that could affect the described procedure is obviously represented by the correct evaluation of the limit elastic resistance of the mild steel, considering the possibility that it may assume different values in tension and compression.

Keeping in mind these warnings, the abacus shown in (Fig. 10) is particularly effective for the rapid preliminary design of the mild and prestressing steel ratio, as well as for the immediate assessment of the self-centering capacity of the connection.

The abacus contains the fundamental parameters involved in the design of the hybrid connections, as later ex- plained, and was obtained in the simplifying assumption of an elastic-perfectly plastic constitutive model, with equal over-strength factors both in tension and in compression, for the mild reinforcements

In the first quadrant of the abacus, the function $m_{r p t}=f(\xi)$ provided by eq. (10) is plotted, after dividing it by the parameter $\psi_{s}=f_{s y} / f_{c}$ for an easier graphical representation.

The second quadrant contains instead the plot of the function $\xi=\xi\left(\rho_{p}\right)$ expressed by eq. (14), which defines the minimum quantity of post-tensioned reinforcement capable to guarantee the required flexural capacity $m_{r p t}$ when the position reached by the neutral axis varies, and the steel remains within the proportionality limit. 


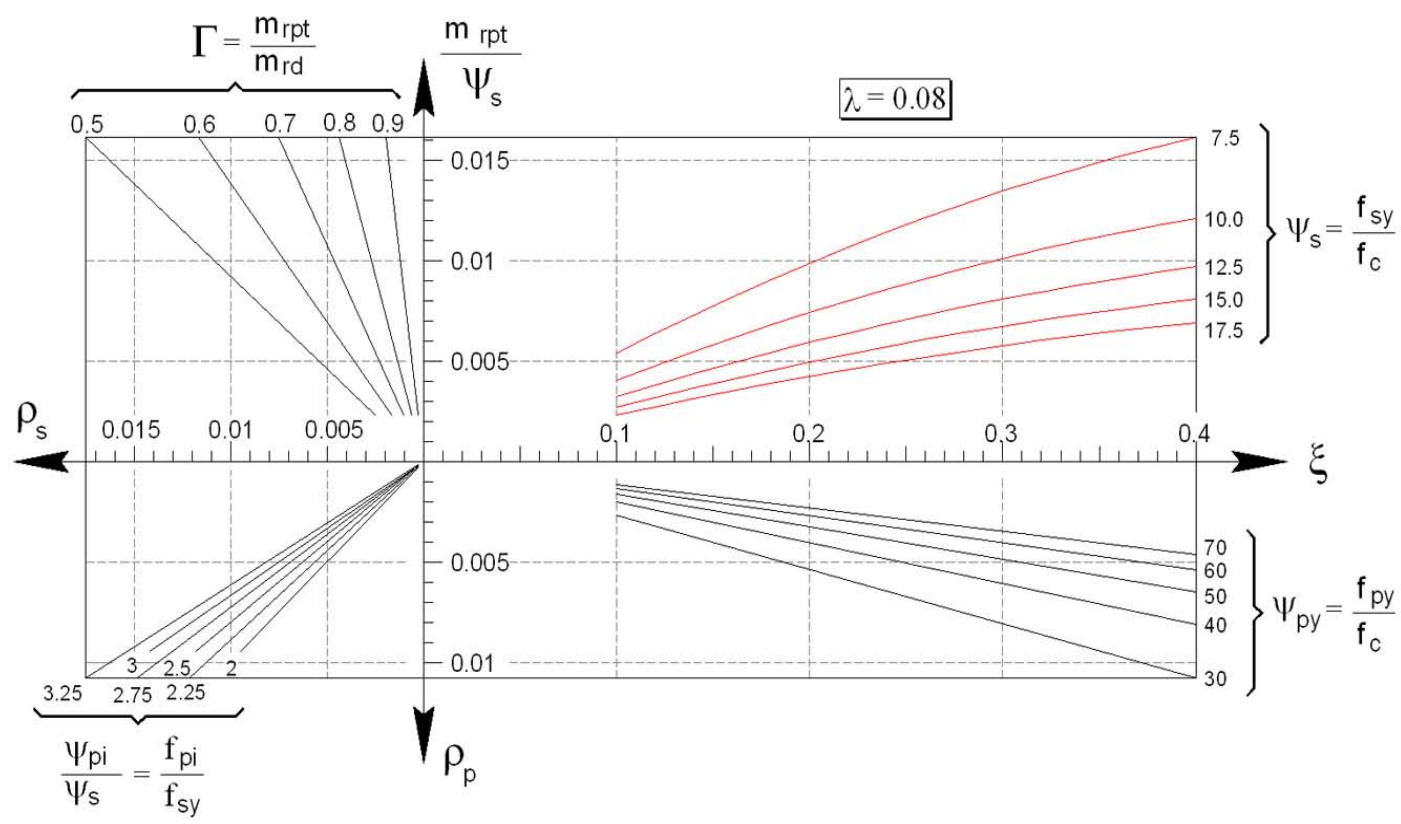

Fig. (10). The abacus for the design of the reinforcements and for the verification of the self-centering capacity hybrid beam-column connections.

In the third quadrant, the amount of mild reinforcement that, after the yielding point, is able to guarantee the flexural capacity $m_{r s}$ as well as the dissipation demand is plotted as a function of the parameter $\Gamma$, according to eq. (15).

Finally, the third quadrant of the abacus shows the plot of the linear equation $\rho_{s}=0.5 \rho_{p} f_{p i} / f_{s}$, which divides the quadrant into two half-space and, recalling eq. (17), allows the immediate appraisal of the system's self-centering capacity. It should be also paid attention to check that the maximum value of the ratio $f_{p i} / f_{s}=\psi_{p i} / \psi_{s}$ satisfies eq. (12), so that the cable remains within the elastic field.

The abacus presented in (Fig. 10) can be a very useful practical tool for the dimensioning beam-column hybrid nodes, by following the procedure presented in (Fig. 11), through a practical example of the application.

It is supposed that the materials and their characteristic mechanical parameters are preliminarily assigned for the connection (concrete class, steel type). Thence, in each quadrant a single curve is extracted from the families contained in the general abacus of (Fig. 10).

Once the design parameters $\psi_{s}, \psi_{p y}, \psi_{p i}, \psi_{E}$ and $\Gamma$ are assigned, together with the design resistant moment $m_{r d}$, the ordinate of point A, given by $m_{r p t} / \psi_{s}=\Gamma \cdot m_{r d} / \psi_{s}$ is immediately identified. Starting from point $A$, the path $A B C D$ is followed until the proper curve $\psi_{s}(\xi)$ is intersected (the selection of the curve, as previously remarked, is governed by the materials' properties), and this determines the area of the post-tension reinforcement $A_{p}=\bar{\rho}_{p} \cdot b d$ which, at the limit of proportionality, provides the moment $m_{r p t}=\Gamma \cdot m_{r d}$ together with the stressed concrete under limit conditions.
Always starting from point $\mathrm{A}$, but proceeding in vertical along the path $\mathrm{AEF}$, the intersection found with the horizontal axis $\rho_{s}$ corresponds to the mild reinforcement ratio which, at the yielding, ensures the contribution $m_{r s}=(1-\Gamma) \cdot m_{r d}$, i.e. it is possible to dimension the ordinary reinforcement $A_{s}=A_{s}^{\prime}=\bar{\rho}_{s} \cdot b d$.

The assessment of the self-centering capacity of the system is performed by checking if the point $\mathrm{G}\left(\bar{\rho}_{s}, \bar{\rho}_{p}\right)$ lying in the $3^{\text {rd }}$ quadrant falls within the area shaded in (Fig. 11), that is characterised by the condition expressed by eq. (16): $\bar{\rho}_{s} \leq 1 / 2 \bar{\rho}_{p}\left(\psi_{p i} / \psi_{s}\right)_{\max }$. The ratio $\left(\psi_{p i} / \psi_{s}\right)_{\max }$ can be easily calculated from eq. (12), after analytically obtaining $\bar{\beta}$ from eq. (11), or, in a quicker way, graphically determining it from the abacus in (Fig. 8).

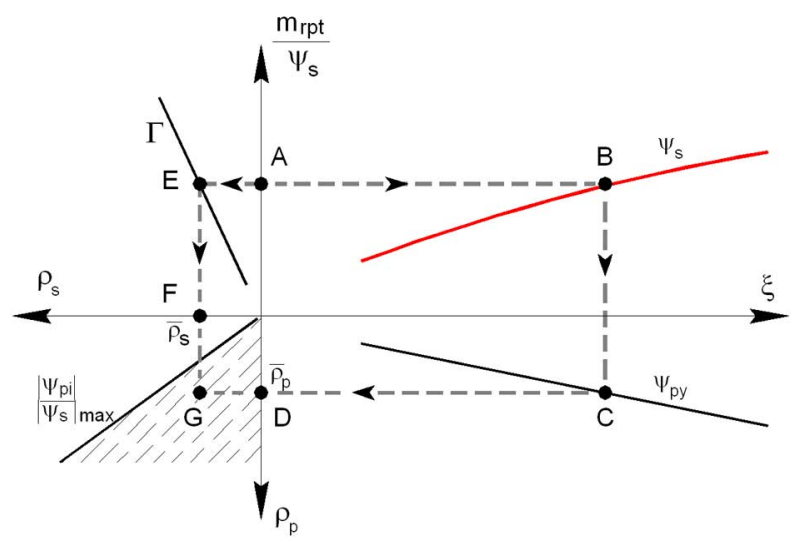

Fig. (11). Example of the use of the design 4-way abacus of Figure 10 . 


\section{CONCLUSION}

The aim of the proposed procedure is to provide a simple tool for the preliminary design of the main parameters governing the resistance of an hybrid connection, under some simplifying - but reasonable - assumptions: rectangular sections with symmetrical mild reinforcements and centered post-tension.

In particular, a non-dimensional 4-way abacus has been plotted, which allows, through an immediate graphical approach, the calculation of the following parameters:

- steel ratio both for the mild reinforcement steel and for the post-tensioned cables;

- tensile force to be applied to the unbonded tendons in order to guarantee an elastic behavior.

Even if the abacus was built under the assumption that the mild steel is yielded, whereas the post-tensioned cables' strain do not exceed the limit of proportionality, the use of non-dimensional parameters in the abacus easily enables to change the above mentioned hypotheses.

For instance, in order to take into account the presence of hardening in the constitutive behavior conditions the ordinary reinforcement, or to limit the maximum strain in the cables, suitable reduced values of the normalized factors $\psi_{s}=f_{s y} / f_{c}$ and $\psi_{p y}=f_{p y} / f_{c}$ can be introduced in the abacus.

\section{CONFLICT OF INTEREST}

The author(s) confirm that this article content has no conflicts of interest.

\section{ACKNOWLEDGEMENT}

The research presented in this article was partially funded by the Italian Department of Civil Protection, Project ReLUIS-DPC 2010-2013.

\section{REFERENCES}

[1] CEN. Eurocode 8: Design provisions for earthquake resistance of structures - Part 1: General rules, seismic actions and rules for buildings. European Committee for Standardisation EN1998-1, Brussels, Belgium, 2005.

[2] DM 14/01/08 "Norme tecniche per le costruzioni" - NTC2008, G.U. n. 29., 4th February 2008; Roma; Italy (in italian). 2008.

[3] ACI Committee 318 - Structural Building Code: Building code requirements for structural concrete (ACI 318m-08) and Commentary. ACI, June 2008.

[4] M.J.N. Priestley, and J.T. Tao, "Seismic Response of Precast Concrete Frame with Partially Debonded Tendons", PCI Journal, vol. 38 , no. 1 , pp. $58-69,1993$.

[5] W.C. Stone, G.S. Cheok, and J.F. Stanton, "A Hybrid Reinforced Precast Frame for Seismic Region”, PCI Journal, vol. 42, no. 2, pp. 20-32, 1997.

[6] M.J.N. Priestley, S. Srithara, J.R. Conley, and S. Pampanin, "Preliminary Results and Conclusions from the PRESSS Five-Story Precast Concrete Test Building", PCI Journal, vol. 44, no. 6, pp. 42-67, 1999.

[7] G. Uva, F. Porco, A. Fiore, and M. Mezzina, "Proposal of a methodology of in-situ concrete tests and improving the estimate of the compressive strength", Construction and Building Materials, vol. 38 , no. 1, pp. 72-83, 2013.

[8] A. Fiore, F. Porco, G. Uva, and M. Mezzina, "On the dispersion of data collected by in situ diagnostic of the existing concrete", Construction and Building Materials, vol. 47, pp. 208-217, 2013.

[9] F. Porco, and G. Uva, "Assessment of the reliability of Structural Concretes During Execution Phases", In: M. Papadrakakis, V. Papadopoulos, V. Plevris, Eds. COMPDYN 2013-4th ECCOMAS Thematic Conference on Computational Methods in Structural Dynamics and Earthquake Engineering, Kos Island: Greece, June 12$14 ; 2013$.

[10] M.J.N. Priestley, G.M. Calvi, and M.J. Kowalsky, Displacementbased seismic design of structures, IUSS Press: Pavia, Italy, 2007.

[11] M.J.N. Priestley, "Direct Displacement-Based Design of Precast/Prestressed Concrete Buildings", PCI Journal, vol. 47, no. 6 , pp. 66-79, 2002.

[12] M.J.N. Priestley, Myths and Fallacies in Earthquake Engineering, IUSS Press: Pavia Italy, 2003.

[13] Federation internationale du beton, fib Bulletin n. 27 "Seismic design of precast concrete building structures", ISBN 978-288394-066-6, January 2004.

[14] M. Mezzina, D. Raffaele, and A. Vitone, Eds. "Teoria e Pratica delle costruzioni in cemento armato - Vol. I Dalla concezione strutturale alle verifiche agli stati limite", DeAgostini Scuola S.p.A., 2007 (in italian)

\begin{tabular}{lcc}
\hline Received: May 20, 2013 & Revised: July 31, 2013 Accepted: August 03, 2013 \\
(C) Porco et al.; Licensee Bentham Open. &
\end{tabular}

This is an open access article licensed under the terms of the Creative Commons Attribution Non-Commercial License (http://creativecommons.org/licenses/by-nc/3.0/) which permits unrestricted, non-commercial use, distribution and reproduction in any medium, provided the work is properly cited. 